NICOLAS BRUCKER

ORCID: 0000-0002-1510-6161

Université de Lorraine, Centre Écritures

nicolas.brucker@univ-lorraine.fr

\title{
LA QUERELLE DU LUXE SUR LES PLANCHES : LE DISSIPATEUR DE DU RIVET
}

La querelle du luxe s'est d'abord exercée dans le domaine des idées, économiques d'abord, morales et politiques ensuite ${ }^{1}$. À la suite de la Fable des abeilles, dont la diffusion dans sa traduction française a concouru à l'éclosion de la polémique au milieu des années 1720, elle rebondit en 1734 avec l'Essai politique sur le commerce de Melon, dont le poème du Mondain de Voltaire donne une version provocatrice et légère. L'article de Saint-Lambert dans l'Encyclopédie, qui marque dans l'histoire de la querelle un point de maturité, en propose un premier bilan et en indique une possible résolution. Il donne du luxe une définition qui tend à se distancier de l'acception exclusivement morale dans laquelle on enfermait le mot jusqu'alors, et qui préfigure l'usage moderne par lequel on désigne aujourd'hui une noble industrie, où s'illustrent un savoir-faire et une tradition, et où se sont formées quelques-unes des plus grosses fortunes mondiales. La pièce dont nous proposons ici l'étude, Le Dissipateur du jésuite Nicolas Gabriel Papillon du Rivet ${ }^{2}$, se situe précisément en ce point où le luxe est encore le lieu d'exercice d'un discours moral, mais sans pourtant viser à sa complète éradication : les

1 D. Margairaz, «Luxe », [dans :] Dictionnaire européen des Lumières, M. Delon (dir.), Presses universitaires de France, Paris 1997, pp. 762-765 ; P. Retat, «Luxe », Dix-huitième Siècle 26, 1994, pp. 79-88.

2 N.G. Papillon du Rivet (1717-1782) est l'auteur de poèmes de circonstance, en latin et en français, et de sermons prêchés lors du Carême de 1760. On lui attribue trois comédies jouées à Louis-le-Grand : Les Pygmées (1745), Le Dissipateur (1746), L'École des jeunes militaires (1748). Voir J.-P. Migne, Collection intégrale et universelle des orateurs sacrés, t. 59, Paris 1844, p. 1178. 
débats d'ordre économique ont au moins produit dans l'opinion ce résultat qu'on n'admet plus sans examen la disqualification pure et simple du mot comme de la chose. Comme les passions qu'il alimente, le luxe serait en soi indifférent : seul son excès est condamnable. L'idéal de mesure, caractéristique de l'éthique des jésuites, s'applique aussi aux vanités du monde : la jouissance des biens terrestres n'est à proscrire que dans le cas où elle perturbe la règle qui assigne à chacun sa juste place dans la société.

\section{LUXE ET DISTINCTION}

La comédie jésuite fait au début du XVIII ${ }^{\mathrm{e}}$ siècle de rapides progrès, grâce au père du Cerceau, qui s'est employé à donner aux petits pensionnaires du collège Louis-le-Grand, âgés de 8 à 12 ans, un répertoire où ils pussent s'exercer à la fois à l'art de bien dire, condition de la réussite pour un gentilhomme appelé à paraître dans le monde, et à retrouver, sur un mode ludique, les connaissances, notamment littéraires, que leurs études leur ont permis d'acquérir ${ }^{3}$. À côté des pièces en latin, qui est la langue d'étude et de communication ordinaire au collège, les pièces en français font peu à peu leur entrée, pour les plus jeunes élèves d'abord, et dans le genre plus léger de la comédie ou du divertissement, pour finalement s'étendre au cours des années 1740 à l'ensemble du répertoire. La maîtrise de la langue française est alors reconnue par les maîtres jésuites comme un objectif pédagogique à part entière.

Comme on peut s'y attendre, l'usage du français coïncide avec une présence renforcée des sujets d'actualité. La comédie de mœurs fait entrer sur la scène des collèges les préoccupations du moment. Aux références historiques ou mythologiques dont le théâtre de collège est rempli, se mêlent des échos des journaux du temps, événements ou comportements. L'éducation morale s'exerce alors sous couvert d'une satire feutrée des modes, tendances et préjugés. La référence à la comédie moliéresque est constante, avec des ouvertures à d'autres auteurs, comme Regnard ou Destouches. Le titre du Dissipateur n'est pas sans rappeler la comédie du même nom que ce dernier fit paraître en 1736, et qui ne sera représentée pour la première fois qu'en 1753. En dehors du titre et du caractère central qu'il définit, il n'y a pourtant guère de rapport entre les deux pièces, sinon une volonté de renouveler la taxonomie des caractères héritée de Théophraste.

Si l'on reprend la distinction que fait Saint-Lambert dans l'Encyclopédie entre « luxe de distinction » et « luxe de bienséance », on comprendra, à la lecture de la pièce, que c'est de la première catégorie que Du Rivet fait la critique. Sur un

Le manuscrit de la pièce, inédite, est conservé à la Bibliothèque de la Ville de Paris, ms CP 4317 [copie de 1773, 124 p.].

3 P. Peyronnet, «Le théâtre d'éducation des jésuites », Dix-huitième Siècle 8, 1976, pp. 107120 ; sur la comédie pp. 112-113. 
mode linéaire, défilent les principales tentations offertes à l'aristocrate du premier XVIII ${ }^{\mathrm{e}}$ siècle. Le catalogue donne ainsi un échantillon de ce qui est le plus prisé alors. Les références aux idées et réalités du temps confirment l'impression d'une pièce qui cherche à coller de très près à l'actualité.

Ainsi, au chapitre de l'architecture, qui est la première des tentations qui vient assaillir Floridor, il faut remarquer que durant les années 1720 le faubourg Saint-Germain s'impose comme le quartier à la mode. Les aristocrates quittent leurs vieux et insalubres hôtels du Marais pour s'installer dans ce secteur aéré où s'érigent sur de vastes parcelles de somptueux palais. Parmi les exemples qui nous restent de ces élégantes demeures, l'hôtel de Matignon fait figure de fleuron. Son premier propriétaire, sorte de Floridor, dut vendre sa maison avant de la voir achevée, la démesure de son projet ayant entraîné sa ruine ${ }^{4}$. On songe à un caractère de La Bruyère qui en construisant sa maison «s'est noyé de dettes pour la porter à ce degré de beauté où elle vous ravit $»^{5}$.

L'hôtel aristocratique est par excellence le lieu de la distinction : l'architecture y présente à l'extérieur les symboles de la puissance de son occupant, et à l'intérieur le raffinement des espaces de réception ${ }^{6}$. Le soin que Floridor met à édifier une demeure qui réponde aux derniers critères du goût confirme l'importance symbolique de l'habitat. Il s'aperçoit, trop tard, que l'architecte n'a pas prévu de terrasse. «Ni terrasse, ni vue », s'exclame-t-il alors à plusieurs reprises, marquant avec une emphase toute comique une indignation qui est celle d'un amateur éclairé. Mais l'hôtel n'est pas qu'une représentation symbolique du rang tenu par son occupant : c'est aussi un lieu d'interactions économiques, comme l'a montré Natacha Coquery ${ }^{7}$. Certes il existe déjà à l'époque d'élégantes boutiques propres à recevoir la clientèle chic. L'habitude de se rendre au domicile des particuliers pour y proposer ses services se poursuit toutefois tout au long du siècle. Marchands, revendeuses à la toilette, artisans de toutes sortes défilent ainsi dans les salons dorés des riches propriétaires. Floridor reçoit ainsi plusieurs marchands, avant de recevoir ses débiteurs, et pour finir ses créanciers.

Le poste de dépense le plus important, après l'entretien de la maison, l'emploi de la domesticité et les frais de réception, revient à la musique. Au début de la scène 6 de l'acte I, Floridor accueille des musiciens nouvellement arrivés de Rome et qui remplacent l'orchestre français dont il vient de se séparer. C'est là

${ }^{4}$ L. Hautecœur, Histoire de l'Architecture classique en France, t. III : Première moitié $d u$ XVIII siècle. Le style Louis XV, A. Picard et J. Picard (dir.), Paris 1950 ; A. Gady, Les Hôtels particuliers de Paris du Moyen Âge à la Belle Époque, Parigramme, Paris 2011, pp. 206-207.

${ }^{5}$ La Bruyère, Les Caractères, chap. VI : « Des Biens de Fortune », § 79, E. Bury (dir.), Le Livre de Poche, Paris 1995, p. 288.

${ }^{6}$ On se reportera aux analyses classiques de Norbert Elias sur l'hôtel particulier, notamment la division et la fonction des espaces de réception (La Société de cour, P. Kamnitzer et J. Étoré (trad.), coll. « Champs-Essais », Flammarion, Paris 1985, pp. 30-32).

${ }^{7}$ N. Coquery, L'hôtel aristocratique. Le marché du luxe à Paris au XVIII siècle, Publications de la Sorbonne, Paris 1998, p. 19. 
le reflet très exact de l'influence exercée par le baroque italien en France dans la première moitié du siècle, et du succès que remportent alors Corelli puis Locatelli. Aussi le Neveu de Rameau pourra-t-il vingt ans plus tard se moquer de la vogue italienne qui prétendit introduire en France la « nouvelle musique ${ }^{8}$. C'est précisément à cette nouvelle musique que Floridor ouvre tout grand les portes. Mais ce revirement n'est pas du goût de son ami le Chevalier qui, affichant un conservatisme rétrograde, avoue ne pas apprécier les sophistications de la musique italienne, le style contrapuntique ni la technicité du bel canto. Pour lui tout cela n'est qu'un « bruit » contraire à la nature, donc incapable d'éveiller en lui la moindre émotion. Il lui oppose le plain-chant français. Ce n'est là qu'une étape d'une contradiction esthétique qui connaîtra dans la suite du siècle plusieurs rebondissements, de la Querelle des Bouffons à la bataille rangée qui opposa gluckistes et piccinistes. Floridor accuse son ami de «mauvais goût ». Voilà le mot lâché. Avoir de l'esprit, c'est-à-dire la faculté de discerner le bon du mauvais en matière esthétique, est la première marque de distinction. La naissance même ne saurait justifier un noble qui manquerait à cette exigence. Aussi est-ce la grande affaire de l'éducation, et il n'est pas indifférent que la question soit soulevée dans une comédie de collège. Floridor exprime par ses choix de dépense la sûreté de son goût et l'infaillibilité de son esprit : ses achats, qu'on pourrait attribuer à une forme de folie, sont en réalité guidés par une " raison ingénieuse ${ }^{9}$. En dehors de la scène où il s'oppose au Chevalier sur le goût musical, il signale la supériorité de son esprit en deux autres endroits : la négociation avec M. Canichon, où il montre un savoir consommé en matière de race canine (II, 4) ; et l'échange avec La Palabre (II, 13), où il prouve sa connaissance de la poésie.

Floridor n'est donc pas un autre Jourdain : s'il est parfois la dupe de son amour-propre, il se signale en revanche par son aptitude à bien juger, et montre un sens supérieur qui correspond à ses prétentions de rang et de sang. Il n'a rien d'un imposteur : son génie personnel est au niveau de ses ambitions sociales. Il n'est pas fou non plus. Sa marotte est raisonnée. Ainsi la légèreté avec laquelle il se laisse convaincre d'être en possession des plus rares antiquités, alors qu'on ne lui vend que des " colifichets », ne prouve pas tant sa bêtise ou sa naïveté que la hâte qu'il a de constituer au plus tôt un cabinet de curiosité, qui doit dans sa maison tenir lieu de pièce maîtresse, destinée à donner, sur un mode visuel, un échantillon de l'étendue et de la variété de ses connaissances. Très sensément, il objecte à Dorante, déterminé à lui vendre le talon de la botte de Charlemagne, qu'en ce temps-là on n'était pas chaussé à la mode d'aujourd'hui (II, 7). Il ne cherche pourtant pas à pousser plus loin la discussion : il lui suffit d'avoir obtenu une réponse vraisemblable pour s'en satisfaire, tant il lui importe de compléter sa

8 D. Diderot, Le Neveu de Rameau, J. Fabre (dir.), Droz, Genève 1963, p. 81.

9 Voltaire, article « Esprit», [dans :] Euvres complètes, J. Vercruysse (dir.), vol. 33 : Euvres alphabétiques, Voltaire Foundation, Oxford 1987, p. 51. 
collection, et d'y faire figurer en bonne place une antiquité prestigieuse. D'ailleurs ne déclare-t-il pas, aussitôt sa décision prise, sa résolution de faire dorer ce talon? Comme Midas qui transforme tout ce qu'il touche en or, Floridor entend donner valeur à ce qu'il possède par le seul fait de posséder. L'esprit par lequel il a décidé un achat confère à l'objet un prix qu'il n'avait pas originellement. Ce principe d'élection, appliqué à des objets, aurait pu s'appliquer à des personnes, une maîtresse par exemple, sur laquelle le marquis aurait jeté son dévolu, révélant ainsi son goût en matière de beauté féminine et de maîtrise des codes de la galanterie. Mais nous sommes dans une comédie de collège, où toute allusion de cet ordre est strictement proscrite.

C'est encore dans le sens de la métaphore aurifère qu'il faut lire la scène qui fait apparaître le personnage de l'alchimiste (III, 7). Il serait facile d'en conclure une fois de plus à la folie de Floridor. En réalité l'or que lui promet Ducreuset n'est pas tant or à gagner qu'à dépenser. Il lui promet en effet « Plus d'or et de bon or qu'on n'en a dépensé, / Depuis que de notre art la vogue a commencé ». Cet or qui l'obsède si bien qu'il en orne jusqu'à son nom est métaphorique de son désir. Il importe peu dans sa matérialité. Changer le plomb en or, c'est comme changer le talon d'une botte hors d'usage en une vénérable relique : l'opération de transformation correspond à la métamorphose que le désir fait subir aux réalités du monde. Par l'élection qu'il leur fait subir, l'esprit de Floridor les ennoblit, il les dore au propre et au figuré, autrement dit il les sublime.

Il faut cependant nuancer ce qui vient d'être dit, car il est évident que le désir de Floridor ne s'exerce pas seul, mais qu'il trouve à s'exprimer au sein d'une triangulation, dont le troisième terme est tenu par le goût dominant, celui de son monde. Bâtiment, musique, curiosités, chiens et chevaux, tout cela relève d'un goût aristocratique qui l'identifie culturellement à l'élite parisienne. L'émulation, bien présente tout au long de la pièce, est essentielle au processus d'objectivation. Le Baron est là pour faire honte à Floridor de ne pas posséder les meilleurs chiens courants (II, 4) : il pique son orgueil, provoque son amour-propre. Lui-même trouve un intérêt sensible à cette constante surenchère : il en retirera des dividendes sonnants et trébuchants. La titulature des personnages, qui correspond, selon une convention familière au lecteur de romans ou au spectateur de comédies, à une hiérarchie dans le pouvoir et dans le mérite, place Floridor dans une situation médiane : marquis, il est inférieur au Duc, chez qui il va jouer à la fin de l'acte I, mais il est supérieur au Baron et au Chevalier qui viennent hanter sa maison. Référence pour les uns, il prend lui-même son modèle chez d'autres personnages que nous ne voyons pas, mais qui sont évoqués pour signifier un principe de chaînage, caractéristique d'une organisation sociale structurée en rangs, conditions et fortunes : A imite $\mathrm{B}$ qui imite $\mathrm{C}$, etc., jusqu'au roi, qui, dans la société de cour, demeure la référence ultime. 


\section{UN LIEU D'AFFRONTEMENT}

Si la folle émulation dont le luxe est le vecteur touche un caractère, elle affecte en réalité la société tout entière. L'ordre social repose désormais sur une économie des signes du pouvoir. La comédie de caractère cache en fait une comédie de mœurs. L'affrontement que représente la pièce est donc généralisé, mais l'action dramatique l'illustre par des options scéniques qui privilégient une série de face-à-face. Nous pourrions parler de figures affrontées, comme ces effigies d'empereurs romains qu'on voit gravées sur les pièces de monnaie antiques, ou comme les estampes représentant deux acteurs de la comédie italienne engagés dans une violente altercation. Front contre front, le corps arqué, tendu dans un mouvement d'agressivité, comprise au sens étymologique d'adgredi, ceux-ci traduisent par le geste et par la parole l'intensité du conflit qui les anime. Si le ton de la comédie de mœurs requiert plus de distance et de médiation dans les rapports humains, il n'en est pas moins vrai que plusieurs scènes du Dissipateur actualisent par la situation, le dialogue et le jeu, des cas d'affrontement.

L'affrontement le plus frappant est celui qui oppose à plusieurs reprises les deux frères. Par comparaison avec son cadet Floridor, Phronime fait figure de sage. Âgé de soixante ans, c'est un Caton moderne : censeur de la frivolité, satiriste de la folie des hommes, observateur qui laisse échapper des commentaires désabusés, mais aussi moralisateur qui assène ses remontrances à son frère. Il est aussi sa mauvaise conscience : il vient lui rappeler l'esclavage dont est victime son fils Eugène depuis de longs mois, tandis que son autre fils Valère attend en vain un brevet de régiment qui lui permettrait de s'établir. Guidés par des philosophies antagonistes, qui les mènent dans des trajectoires de vie opposées, les deux frères ne sauraient se comprendre : leurs dialogues tournent court. À travers eux s'expriment deux conceptions de l'homme et de la place qu'il doit tenir dans la société. Derrière le rigorisme de Phronime se dessine l'éthique fénelonienne d'une noblesse qui tend à défendre un idéal de sobriété dans le mode de vie, un attachement aux traditions ancestrales, au nom et à la terre ${ }^{10}$.

Dans un premier temps l'affrontement paraît tourner à avantage de Phronime, dont les remarques frappent par leur bon sens. Il semble se faire l'écho d'une sagesse immémoriale, celle de la condamnation des plaisirs inutiles. Le stoïcisme de Sénèque dans le De Vita beata parle par sa bouche : il dénonce ainsi la vie aliénée par la poursuite des faux biens. On peut aussi voir en lui la traduction d'une morale bourgeoise qui privilégie l'épargne à la dépense ostentatoire, l'investissement à la dilapidation des biens. Phronime face à Floridor, c'est en somme l'impatience de $\mathrm{M}^{\mathrm{me}}$ Jourdain devant les fantaisies de son mari. On peut enfin lire dans ce duel le conflit entre une loi ancestrale qui protège la famille, en particulier sous l'angle de la transmission du patrimoine, des écarts de conduite du mauvais sujet, qui

10 F.-X. Cuche, Une pensée sociale catholique. Fleury, La Bruyère, Fénelon, Le Cerf, Paris 1991, pp. 301-303. 
mange sa fortune, fait des dettes, expose son nom à l'infamie. Les registres de la Bastille sont pleins de ces jeunes imprudents qu'un conseil de famille a su réduire au silence, par une lettre de cachet qui leur aura valu quelques semaines de détention, généralement suivies d'une déportation dans les îles ${ }^{11}$. La tranquillité et l'ordre des familles sont à ce prix.

L'affrontement n'est pourtant pas binaire. La pièce est conçue de telle sorte que les caractères se révèlent peu à peu au cours de l'action, laissant voir la complexité de chaque personnage, et l'impossibilité de l'identifier à un modèle unique. De Phronime, on apprend qu'il déteste la musique, ce qui le rend suspect : son ton brusque, sa mine renfrognée l'assimilent à un gentilhomme campagnard, d'une rusticité rugueuse, tout éloignée des bonnes manières de la cour. Il ignore ce qu'on doit à l'honnêteté et à la bienséance. C'est ce dernier mot qu'emploie SaintLambert pour qualifier ce qu'il considère comme un luxe acceptable : le « luxe de bienséance » est non seulement permis, mais il est nécessaire aux peuples civilisés. Mais c'est surtout à la fin que Phronime révèle au grand jour son inhumanité : à la nouvelle de l'arrestation de son frère, il ne cache pas sa satisfaction. Il souhaite même que sa détention se prolonge un peu, de façon à ce que la leçon soit complète (III, 18). Valère s'étonne que son oncle puisse tenir un propos si dur, tandis qu'Eugène s'emploie à faire émanciper son père sans délai.

La disqualification de Phronime interdit de voir en lui une alternative crédible à la manie dissipatrice de Floridor. Elle interdit aussi de lire la pièce comme un simple apologue, à la manière de certaines des comédies du père Du Cerceau. Euloge, ou le danger des richesses, comédie représentée par les petits pensionnaires du collège Louis-le-Grand le 2 juin 1725, se passe à Constantinople, durant le règne des empereurs Justin et Justinien. Elle met en scène Euloge, qui de pauvre artisan devient grand officier du palais, mais qui, après avoir rudement éconduit son ancien ami Arsène, solitaire retiré dans la Thébaïde, retombe dans la misère, avant d'être obligé d'avoir recours à ce même ami, qui lui prodigue les secours espérés. La morale, pourtant transparente, est explicitée : « combien les richesses sont un péril dangereux pour la vertu ». Phronime n'est pas Arsène, et la comédie de Du Rivet est plus complexe que celle de Du Cerceau, qui n'est au fond qu'une comédie-proverbe.

De son côté Floridor surprend également : à l'excentricité dont il fait montre dans la scène liminaire lorsqu'il répète machinalement " ni terrasse, ni vue ", font suite le ton très sensé avec lequel il interroge M. Canichon afin d'avoir du couple de chiens que celui-ci veut lui vendre une idée précise, le jugement parfaitement fondé que lui inspirent les vers de La Palabre, et surtout les remords qui lui viennent de n'avoir pu trouver la somme qui eût permis la libération d'Eugène. Ces passages nous rendent le personnage sinon sympathique, du moins intéressant. Ses contradictions, qui lui donnent à la fois profondeur et vérité, interdisent

11 A. Farge, M. Foucault (dir.), Le désordre des familles. Lettres de cachet des Archives de la Bastille au XVIII siècle, Gallimard, Paris 1982. 
de voir en lui un simple caractère. Elles lui confèrent la densité et l'autonomie d'un personnage : son existence ne se résout pas au seul antagonisme qui l'oppose à son frère.

C'est ailleurs qu'il faut chercher le mobile de l'action de Floridor. Dans quel combat celui-ci est-il engagé ? La réponse se trouve dans la bouche de La Fleur, le rusé valet, jamais avare de bons mots et de saillies mordantes. Celui-ci, pour le mettre en garde, annonce à son maître qu'il va bientôt devoir « affronte[r] la cage » (III, 11). Il signifie par là que les entrevues qu'il a avec ses créanciers, au cours desquelles il se livre à un savant jeu d'esquive avant de les éconduire successivement, ou, comme c'est le cas face au redoutable Monsieur Griffon, de prendre la fuite, ne le sauveront pas d'un ultime face-à-face, avec l'institution judiciaire cette fois, et que celle-ci ne se montrerait pas si accommodante qu'ont pu l'être ses créanciers. Affronter la cage, c'est aussi se trouver face à un mur, sans possibilité de pouvoir dialoguer, c'est enfin sortir de l'espace de scène : la prison signifie la mort symbolique du personnage.

La Fleur tient tête à son maître en plus d'une scène, au risque de s'attirer ses malédictions. Ce personnage du valet débrouillard et roué, dont le dévouement n'exclut pas le soin de son intérêt personnel, est un des mieux touchés de la pièce : on sent que, dérivé du servus de la comédie latine, il a pris dans le théâtre de Regnard des manières et une sensibilité qui le rendent sympathique. Le jugement que porte sur lui Phronime à la fin de la pièce, invitant son frère à se défier de lui, surprend d'autant plus que le valet l'avait maintes fois mis en garde contre l'issue à laquelle devait le mener sa prodigalité chronique. Les affrontements du valet et du maître tournent court. Ils n'en permettent pas moins de souligner que la distance des classes sociales peut se réduire quand le mérite ne répond pas à la naissance. Contre son attente Floridor n'est pas conduit à la Bastille, là où ses pareils sont normalement « logés » (I, 10), mais dans l'horrible prison du Grand Châtelet, à propos de laquelle courent d'effrayantes légendes sur les cachots noirs et puants où l'on jetait les prisonniers. La détention pour dettes, de droit commun, ne vaut à Floridor nul traitement de faveur : il perd les privilèges qui tiennent à ses titre, rang et qualité. Il n'est plus rien. La moralité de la pièce est aussi à chercher du côté d'une menace d'un chaos social : le luxe mal ordonné ayant conduit au contraire de ce qu'il était censé produire, à savoir la distinction, on sombre dans l'indistinction. À la cage, tout s'égalise.

Mais la cage peut se lire métaphoriquement comme l'enfermement dans lequel l'a peu à peu confiné sa folie dépensière. Il n'est plus de limite que lui-même. Floridor s'isole progressivement : il ne songe plus à ses fils, abandonnés à leur sort, ni à rien qui n'ait un rapport avec les acquisitions dont il a le projet. Sa pensée se déploie dans le cercle étroit des valeurs, prix et catalogues, de ce qu'on peut acheter ou vendre, à quel prix et dans quels délais. En proie à son seul désir, il répète, avec une surprise où perce l'incrédulité, qu'il est le maître de désirer ce qu'il veut. Les séquences successives l'amènent, par l'intermédiaire de personnages 
tentateurs, vrais parasites ou marchands peu scrupuleux, à s'interroger sur ce qu'il veut vraiment. Le dialogue intérieur affleure çà et là dans quelques brefs monologues, où le personnage, ayant perdu l'aplomb qu'il affiche en public, laisse voir à nu son désarroi. On comprend alors que ce qu'il cherche à manifester par ses achats censément distinctifs du rang ou du goût, c'est soi-même : une identité qui ne soit pas réductible à une appartenance sociale.

\section{LE THÉÂTRE, MIROIR DE LA SOCIÉTÉ}

Le théâtre de collège est la vitrine d'un programme, d'une méthode, et des résultats que celle-ci est censée produire. Aussi importe-t-il d'y consacrer tout le temps et l'énergie nécessaires. Charles Rollin, adversaire des jésuites, s'inquiète au contraire de la fatigue que coûtent à leurs auteurs ces pièces, qui en plus les détournent des soins qu'ils devraient employer à l'instruction de leurs élèves ${ }^{12}$. Les deux représentations annuelles (généralement le Mardi gras et à la fête de la SaintLouis) sont l'occasion d'une rencontre entre le petit monde du collège et le grand monde de la société. Le public, dont la nature est précisément décrite par les journaux qui rendent compte des représentations, découvre l'univers clos de ces maisons d'éducation élitistes, où les élèves parlent latin entre eux, où tout est tourné vers les lettres et les arts, la culture de l'esprit et la formation des mœurs. Il découvre cet univers à travers ces fictions théâtrales qu'on lui propose à intervalles réguliers. C'est par là que maîtres et élèves communiquent au dehors la conception qu'ils se font de la société contemporaine, la vision d'un monde à la fois si proche et si lointain. Car les maîtres jésuites, retranchés dans leur cabinet d'étude et dans des références culturelles majoritairement antiques, ne connaissent du monde contemporain que des fragments, qu'ils complètent par la lecture des œuvres anciennes ou modernes auxquelles ils ont eu accès. La scène tend donc au public un miroir à facettes dans lequel se donnent à voir quelques aspects de la société contemporaine à travers le point de vue particulier adopté par les maîtres.

Thorstein Veblen a défini le luxe comme conspicuous consumption, consommation ostentatoire ${ }^{13}$. Sur scène, cette ostentation se traduit par l'action de restituer par une impression visuelle la matérialité des composants du luxe : objets, meubles, instruments de musique, éléments du décor... on imagine aisément quels realia les conditions actuelles de la mise en scène permettent de produire aux yeux du public. Mais le code verbal restant dans le théâtre d'éducation l'élément principal de la dramaturgie, il importe surtout de nommer les choses. Ainsi convoquées à l'existence, celles-ci adviennent instantanément dans l'imagination du spectateur, et d'autant plus facilement qu'il s'agit de réalités familières, dont on parle et qu'on peut avoir

\footnotetext{
12 Cité dans : P. Peyronnet, op. cit., p. 119.

13 N. Elias, op. cit., p. 43.
} 
vues soi-même. Jouée à Paris, au cœur de la ville la plus luxueuse, la pièce jouit d'un retentissement supplémentaire. Par son ton nuancé, elle convient aussi bien aux spectateurs parisiens, sensibles aux allusions à l'actualité, qu'aux spectateurs provinciaux, les parents des élèves qui auront pu faire le déplacement, moins au fait des nouvelles, et plus réceptifs au message moral d'ensemble. La critique du luxe tapageur les touchera d'autant plus qu'ils en seront plus éloignés, et que leurs revenus leur prescriront un mode de vie plus austère.

La comédie ne dénonce donc pas forcément un vice que pourrait partager le public. Le préambule de la pièce avait pris soin d'écarter le modèle de la comédie d'Aristophane, dont la visée morale ambitionnait de corriger les mœurs et de réformer la société. De même, en raillant la figure du pédant, hautain par sa prétention à étaler sa connaissance, indocile aux lois et dédaignant sa patrie pour se revendiquer de la seule hellénité, l'auteur entendait, avant que la pièce commençât, prévenir dans l'esprit du spectateur tout soupçon de moralisme primaire. Celui-ci ne peut donc se méprendre sur les motivations de l'auteur ni sur le dessein de la pièce. Il ne s'agit en aucune façon de condamner le luxe, ni de moraliser l'usage des richesses, mais de mettre en garde contre les excès auxquels expose une dépense inconsidérée. La pédagogie des jésuites se donne des objectifs à la fois théoriques et pratiques. Sur le volet pratique, l'art de ménager son argent occupe une place de choix, comme on le voit par les thèmes des comédies qui sont jouées : Euloge, de Du Cerceau, déjà citée, mais aussi Le Joueur de Porée.

Si le public de la pièce peut être regardé comme un échantillon de la société française, il en est tout autant de la distribution des rôles. Les programmes, heureusement conservés pour les deux représentations à Louis-le-Grand, la première en 1746 et la seconde en 1761, nous renseignent précisément sur le nom, le rang, l'origine de chacun des acteurs. Si l'on considère la création de la pièce, on constate que les différents rangs de la noblesse sont représentés : noblesse princière, noblesse d'épée, noblesse de robe ou de finance. En 1761, des îliens font leur apparition, notamment pour tenir les rôles des voyageurs Dorante et Clitandre. Parmi plusieurs noms d'acteurs appelés à la célébrité, il en est un qui retient notre attention. Gaspard Louis Rouillé d'Orfeuil (1732-1791) tient le rôle de Clitandre en 1746. L'enfant, alors âgé de 14 ans, fils d'un maître des requêtes, fera une brillante carrière dans l'administration royale. On se souvient, notamment par Diderot qui l'a connu à Bourbonne, qu'il fut intendant de Champagne à partir de 1764. Le bel hôtel de la préfecture qu'on peut encore aujourd'hui admirer sur les bords de la Marne à Châlons-en-Champagne est son œuvre, si l'on peut dire, puisque c'est lui qui, en prenant possession de sa charge, décida de modifier les plans du chantier alors en cours ${ }^{14}$. On imagine aisément ses éclats de voix indignés en apprenant que n'étaient prévues « ni terrasse, ni vue ». L'une

14 P. Lavedan, J. Hugueney, P. Henrat, L'urbanisme à l'époque moderne. XVIe-XVIII siècles, Droz, Genève 1982, p. 152. 
et l'autre furent introduites, ce qui ne contribua pas à faire baisser la note d'un chantier déjà pharaonique. Sans doute n'avait-il pas bien profité de la leçon de son maître Du Rivet, ou pas bien compris quelle morale la pièce dans laquelle il avait joué était censée défendre. Car, si l'on en croit la chronique mondaine, il fut dans son particulier maladivement dépensier. Cet amateur de soupers fins et de galante compagnie s'endetta au point de priver ses héritiers de toute succession. On voit qu'il aurait pu jouer Floridor : c'est le rôle qu'il tint durant sa vie, qu'il ne finit pas en prison, comme son modèle, mais dans le plus complet dénuement.

Plus intéressant encore, cet ami des philosophes, esprit libéral et ouvert aux réformes, a apporté sa contribution à la querelle du luxe, arrivée dans sa phase finale. Il exprime un point de vue radical, qui feint d'ignorer les arguments mercantilistes, et manifeste un parti pris foncièrement moral. L'Alambic moral, paru en 1773 , définit le luxe comme « l'habitude du superflu $»^{15}$. Le luxe est le symptôme de la décadence d'une société. « Je n'ai jamais pu concevoir comment des auteurs qui voient bien, qui ont réellement de l'esprit, des connaissances, et sont capables de réflexion, peuvent croire, et osent soutenir que le luxe est nécessaire et utile ${ }^{16}$, s'étonne ailleurs l'auteur, qui fait l'énumération de ses conséquences néfastes, et conclut de là « qu'il est nécessaire et même indispensable de détruire entièrement le luxe ». À l'article « DÉPENSE », il prend le soin de distinguer les besoins réels des besoins d'imagination, qui peu à peu deviennent des besoins d'habitude, pour inviter à retrancher les premiers du budget de la maison, qui s'en trouvera alors accru. Il fait mine d'ignorer la fonction de distinction attachée au luxe : « belle chimère ! quelle gloire peuvent acquérir tous ces prétendus grands qui se ruinent... ${ }^{17}$. Il met enfin en garde contre les conséquences économiques d'une consommation effrénée : la ruine des particuliers signifie la ruine de l'État.

Marqué par la pensée des physiocrates, qui sur le sujet s'impose au cours des années 1750 , le point de vue de d'Orfeuil est aussi l'exact reflet des positions affichées par Turgot dans ses Réflexions sur la formation et la distribution des richesses (1766). Le luxe, qui est également défini comme une « habitude», et en tant que tel étranger à la raison, réduit le capital disponible et détourne le numéraire d'un investissement productif. Les dépenses superflues sont en définitive préjudiciables à la prospérité de la nation. Le luxe, loin d'enrichir, appauvrit. Cette position, antithétique du point de vue libéral dominant dans la première moitié du siècle, est révélatrice d'un changement d'attitude des élites. Le luxe n'est plus un facteur de distinction. La société de cour telle que décrite par Norbert

15 G.L. Rouillé d'Orfeuil, L'Alambic moral, ou Analyse raisonnée de tout ce qui a rapport à l'homme, par l'Ami des Français, [s. 1.] 1773, p. 385.

16 G.L. Rouillé d'Orfeuil, L'Alambic des loix, ou Observations de l'ami des Français sur l'homme et sur les loix, [s. 1.] 1773, p. 49.

17 G.L. Rouillé d'Orfeuil, L'Alambic moral..., p. 194. 
Elias ${ }^{18}$ n'existe plus ; l'ostentation aristocratique est devenue incompréhensible. La France du Dissipateur n'est plus la France de Turgot. Le luxe, facteur de distinction et donc agent structurant du corps social, est devenu en vingt ans une puissance désorganisatrice. La pensée bourgeoise a vaincu, et avec elle une économie reposant sur les biens productifs, le capital, l'investissement.

La pédagogie du rire mise en œuvre par les pédagogues jésuites est attentive à ne pas blesser l'amour-propre ni heurter les sensibilités. Elle ne ridiculise pas les caractères, ni ne cherche à soulever un ris moqueur. Car elle cherche à faire penser, à amener le spectateur à opérer un retour sur lui, en l'occurrence sur ses habitudes de dépense, sur la nature du désir qui le pousse à acheter au-delà de ses besoins essentiels. Dans Le Dissipateur de Du Rivet, la querelle du luxe n'est pas abordée de front, mais de biais, à travers le cas d'un particulier, de ses pratiques de consommation et de son aptitude à en rendre compte de manière raisonnée. Derrière le cas individuel, le spectateur reconnaît aisément des positions morales et philosophiques. Il saisit peut-être aussi, au moins confusément, dans l'opposition structurante entre Phronime et Floridor, un clivage qui traverse toute la société dans la conception et l'usage des richesses. De manière plus générale, la comédie rend compte, à son insu cette fois, de la transition d'une étape à l'autre de la querelle, qui elle-même coïncide avec une transformation de la société française dans la place qu'elle assigne à ses élites.

\section{THE LUXURY DEBATE ON STAGE: LE DISSIPATEUR BY DU RIVET}

\section{Summary}

The French Jesuit theatre, which during the $18^{\text {th }}$ century gradually opened to comedy and French-language performances, reflects social news. First performed in May 1746 at the Collège Louis-le-Grand, Le Dissipateur takes place in a luxury debate which runs from Mandeville to Mirabeau throughout the century. This comedy defends the middle ground and invites actors and spectators to settle their expenses on their social condition. It thus accomplishes its educational aim, in accordance with the spirit of Jesuit pedagogy, which consists in preparing pupils to live in society, to know its rules, so as never to be the victims of it.

Key words: comedy, Jesuit, pedagogy, luxury, waste, expense, quarrel, dispute.

18 Norbert Elias a, dans son analyse de la société de cour, marqué les conditions d'une économie aristocratique de l'ostentation, qui règle les dépenses sur les exigences du rang et non sur la gestion rationnelle des revenus. 